crepancies, and this assumes that the planet Saturn is as black as Jupiter in the three wavelength intervals, what does it say about the composition of the rings? The clue here is a fourth band from $3 \cdot 3$ to 4 microns where both Jupiter and Saturn are black; there is no sign of reflected light from the rings of Saturn. According to Johnson, if the rings consist of $\mathrm{H}_{2} \mathrm{O}$ ice they ought to contribute in the $3 \cdot 3$ to 4 micron band, and there ought to be an ice absorption band at 3 microns as well. Johnson suggests that this makes ammonia ice a more likely explanation. The point here is that ammonia ice has been put forward as the explanation of the absorption which makes Jupiter black between 3.3 and 4 microns, and therefore might explain why the rings of Saturn fail to register at these wavelengths.

\section{MANY-BODY PROBLEM}

\section{Pairwise Harmonic Forces}

\section{from a Correspondent}

From a microscopic point of view all problems in physics and chemistry are many-body problems. The problems become interesting when the particles of the system interact with one another, usually via two-body forces. There are very few systems of interacting particles for which exact solutions are known, and so it is always exciting to study a model of interacting particles which is exactly soluble.

Storer (Phys. Rev. Lett., 24, 5; 1970) has given a quantum statistical mechanics treatment of a system of particles interacting through pairwise harmonic oscillator forces. Working in terms of the grand partition function he shows that this interacting system behaves like a system of independent particles moving in an external harmonic well with, however, an important modification which implies a greatly reduced density of states. Not only is the density of states reduced but the spectrum of a system of particles interacting through the pairwise forces is shifted down by 3 $\frac{3}{2} \hbar \omega$ (where $\omega$ is the oscillator frequency) relative to that of a system of independent particles in an external well.

The problem of describing $N$ interacting particles is usually stated in terms of the $N$ coordinates of the particles $x_{1}, x_{2} \ldots x_{N}$. In the absence of external forces the centre of mass of the system, with coordinate $\vec{X}=\frac{1}{N} \sum_{i=1}^{N} x_{i}$, has its motion conserved, that is, if we fix the centre of mass there remain only $(N-1)$ independent coordinates. This distinction between $N$ independent coordinates and $(N-1)$ independent coordinates is lost in a statistical treatment.

It follows immediately from the definition of the centre of mass coordinate that the energy of the system of particles interacting through pairwise harmonic forces is equal to the energy of a system of independent particles moving in an external harmonic well the origin of which is attached to the centre of mass, which itself describes harmonic motion, plus the energy associated with the motion of the centre of mass.

When we consider the full spectrum of the system of independent particles moving in an external harmonic well, many of the configurations will correspond to excited states of the centre of mass motion. If we neglect the centre of mass motion the density of states will be decreased as observed by Storer. Further, because the centre of mass undergoes harmonic motion the zero point energy associated with this will be $\frac{3}{2} \hbar($, and so when the centre of mass motion is ignored the whole spectrum will be shifted down by this amount.

The many-body system in which particles interact through pairwise harmonic forces has frequently been examined in the context of nuclear structure theory and the results quoted here have long been the basis for the standard method used to remove states containing spurious centre of mass motion from nuclear shell-model calculations (Elliott and Skyrme, Proc. Phys. Soc., A232, $561 ; 1955)$.

\section{FRACTURE MECHANICS}

\section{Brittle Fracture by Thermal Shock}

\section{from our Materials Science Correspondent}

ONE of the many obstacles in the path of the design engineer who would like to make use of the high strength of brittle ceramics such as alumina or silicon nitride is their sensitivity to thermal shock. Thermal gradients induced by sudden heating or cooling cannot be relieved by plastic flow (which is why these materials are strong) and so cracks readily form and propagate. Quantitative treatment of this process is difficult and so it is of considerable importance that a unified theory has been published recently by D. P. H. Hasselman (J. Amer. Ceram. Soc., 52, 600; 1969).

Hasselman has considered three distinct stages in thermal shock: the thermal condition required to initiate fracture (assuming a pre-existing population of small Griffith cracks of uniform size), the length of crack which results and the thermal condition required to cause the arrested crack to start propagating again. It turns out that the temperature difference between surface and core-rather surprisingly, it is the differenco and not the gradient that enters the equations-required to initiate fracture is independent of the density of cracks. But it varies slowly with crack size, while the size of crack at which propagation is arrested depends steeply on initial crack size and also on population density. It is particularly noteworthy that there is a particular initial crack size (depending somewhat on the population density) for which the temperature difference to initiate crack propagation is a minimum: that is, as far as initiation is concerned, the material is then at its most sensitive. In these circumstances, however, crack propagation is least favoured; propagation is quasi-static instead of being dynamic. As a result of his calculations (which agree in detail with some careful measurements by Davidge and Tappin of thermal shock behaviour of alumina spheres), Hasselman recommends that, for a given material, sensitivity to thermal shock should be minimized by adjusting the grain size, or population of artificial cracks, so that fracture is easily initiated but unlikely to propagate.

One incidental feature of interest which emerges from the survey is the asymmetry of thermal shock fracture: alumina is much more apt to shatter on 